\title{
A Precise Estimate of the Radius of HD 149026b
}

\section{Philip Nutzman ${ }^{1}$, David Charbonneau ${ }^{1,2}$, Joshua N. Winn ${ }^{3}$, Heather} A. Knutson ${ }^{1}$, Jonathan J. Fortney ${ }^{4}$, Matthew J. Holman ${ }^{1}$, Eric Agol ${ }^{5}$

${ }^{1}$ Harvard-Smithsonian Center for Astrophysics, 60 Garden St., Cambridge, MA 02138 email: pnutzman@cfa.harvard.edu

${ }^{2}$ Alfred P. Sloan Research Fellow

${ }^{3}$ Department of Physics, and Kavli Institute for Astrophysics and Space Research, Massachusetts Institute of Technology, Cambridge, MA 02139, USA

${ }^{4}$ Department of Astronomy and Astrophysics, UCO/Lick Observatory, University of California, Santa Cruz, CA 95064

${ }^{5}$ Department of Astronomy, University of Washington, Box 351580, Seattle, WA 98195

\begin{abstract}
We present Spitzer $8 \mu \mathrm{m}$ transit observations of the extrasolar planet system HD 149026b. At this wavelength, transit light curves are weakly affected by stellar limbdarkening, allowing for a simpler and more accurate determination of planetary parameters. We measure a planet-star radius ratio of $R_{p} / R_{\star}=0.05158 \pm 0.00077$, and in combination with ground-based data and independent constraints on the stellar mass and radius, we derive an orbital inclination of $i=85_{-0.4}^{\circ}{ }_{-0.9}^{\circ}$ and a planet radius of $0.755 \pm 0.040 \mathrm{R}_{\mathrm{J}}$. These measurements further support models in which the planet is greatly enriched in heavy elements.
\end{abstract}

\section{Introduction}

The hot, Saturn-mass exoplanet HD 149026b $\left(M=114 M_{\oplus}\right.$; discovered by Sato et al. 2005) has garnered much attention due to its potential to directly test models of planet formation. The planet's small observed radius for its mass implies that roughly $2 / 3$ of its mass is in the form of heavy elements - more than found in all of the Solar System planets combined. The existence of such a metal-laden planet orbiting a very metal-rich host star $([\mathrm{Fe} / \mathrm{H}]=0.36)$ is a smoking gun for the core-accretion theory (e.g., Pollack et al. 1996), though the immense quantity of heavy elements is still a challenge to canonical models of core accretion. HD $149026 \mathrm{~b}$ has also received notice for its scorching dayside brightness temperature $(2300 \mathrm{~K})$, which is well in excess of its expected equilibrium temperature. The planet may be a standard bearer for the proposed 'pM' class of planets (Fortney et al. 2008), which are characterized by hot stratospheres and large day/night temperature contrasts caused by opacity at visible wavelengths due to gaseous $\mathrm{TiO}$ and VO high in their atmospheres.

Unfortunately, the present fractional uncertainty in the key observable parameter, the planetary radius $R_{p}$, is $7 \%$ (Winn et al. 2008). This study is inspired by the potential of infrared photometry with the Spitzer Space Telescope to reduce this uncertainty. Because of the near absence of stellar-limb darkening in the infrared, transit light curve modeling is greatly simplified and gives results largely independent of assumptions about limbdarkening coefficients. We present Spitzer $8 \mu \mathrm{m}$ observations of the transit of HD 149026. Together with previously published ground-based data, we derive precise constraints on the radius of HD 149026b and other system parameters. $\dagger$

$\dagger$ For a detailed description of our analysis, please consult Nutzman et al. (2008). 


\section{Observations and Analysis}

We observed the UT August 14, 2007 transit of HD 149026 through the IRAC $8 \mu \mathrm{m}$ channel on the Spitzer space telescope (see Figure 1). We obtained 67,008 images in IRAC subarray mode at a $0.4 \mathrm{~s}$ cadence. The data exhibits very low levels of timecorrelated, or "red", noise, as demonstrated by Figure 2. Figure 2 also indicates that, at 15 minute resolution (bins of roughly 2,000 images), the photometric precision dips below $2 \times 10^{-4}$. In order to achieve the strongest possible constraints on the system parameters, we combined our data with previously published ground-based photometry (Sato et al. 2005, Charbonneau et al. 2006, Winn et al. 2008).

We employed Markov Chain Monte Carlo to derive best-fit parameters and uncertainties (see Table 1). We imposed an external constraint on the stellar mass, as derived by Sato et al. (2005). We also employed the empirical $V, K$ relations of Kervella et al. (2004) for the angular diameters of dwarf stars, and combined with the Hipparcos parallax to derive an external constraint on the radius of the star HD 149026.

IRAC $8 \mu \mathrm{m}$ observations exhibit a detector ramp (see Figure 1 and e.g., Knutson et al. 2007). Our MCMC model includes a simultaneous analysis of a ramp model and the transit model, hence our error bars include the resulting correlations and uncertainties. The data, with and without the ramp correction and binned 100:1 are shown in Fig. 1, together with the best-fitting transit model.
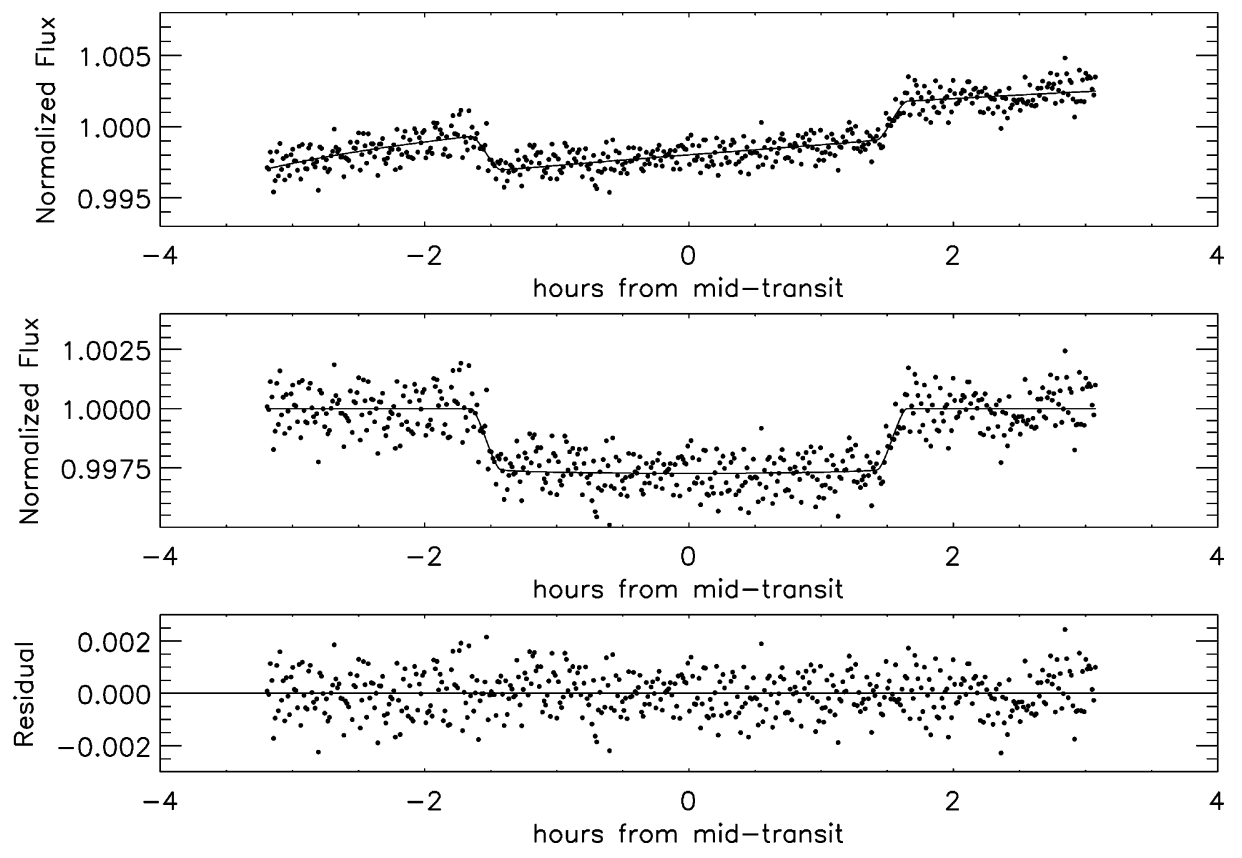

Figure 1. Spitzer transit photometry for HD 149026, with 40 second resolution (bins of 100 images). The top panel displays the raw light curve and exhibits the well-known IRAC $8 \mu \mathrm{m}$ detector ramp (e.g., Knutson et al. 2007). The middle panel displays the ramp-corrected light curve together with the best-fitting transit model. At bottom are the residuals from the best-fit. The root-mean-square residual is only $15 \%$ greater than the expected photon noise. 
Table 1. Best-fit parameters and uncertainties of HD 149026 system properties derived through a Markov Chain Monte Carlo analysis.

\begin{tabular}{lccc}
\hline \multicolumn{4}{c}{$\begin{array}{c}\text { Estimates of the HD 149026 } \\
\text { System Parameters }\end{array}$} \\
\hline Parameter & Median & $15.9^{t h}$ Perc. & $84.1^{\text {th }}$ Perc. \\
\hline$R_{p} / R_{\star}$ & 0.05147 & -0.00077 & +0.00076 \\
$i[\mathrm{deg}]$ & 85.3 & -0.8 & +0.9 \\
$a / R_{\star}$ & 6.20 & -0.25 & +0.28 \\
$R_{p}\left[\mathrm{R}_{J}\right]$ & 0.755 & -0.040 & +0.040 \\
$R_{\star}\left[\mathrm{R}_{\odot}\right]$ & 1.497 & -0.069 & +0.069 \\
\hline
\end{tabular}

\section{Discussion}

We have presented and analyzed Spitzer $8 \mu \mathrm{m}$ transit observations of the HD 149026 system. By incorporating previously published data, and adopting constraints on the stellar mass and radius, we improve the determination of the planetary radius to $R_{p}=$ $0.755 \pm 0.040 \mathrm{R}_{\mathrm{J}}$.

Our measurement reinforces previous findings of the intriguingly small radius of HD 149026b. In comparison, models in which HD 149026b is composed purely of $\mathrm{H} / \mathrm{He}$ require a radius greater than $1.1 \mathrm{R}_{\mathrm{J}}$ (see Figure 3 ).

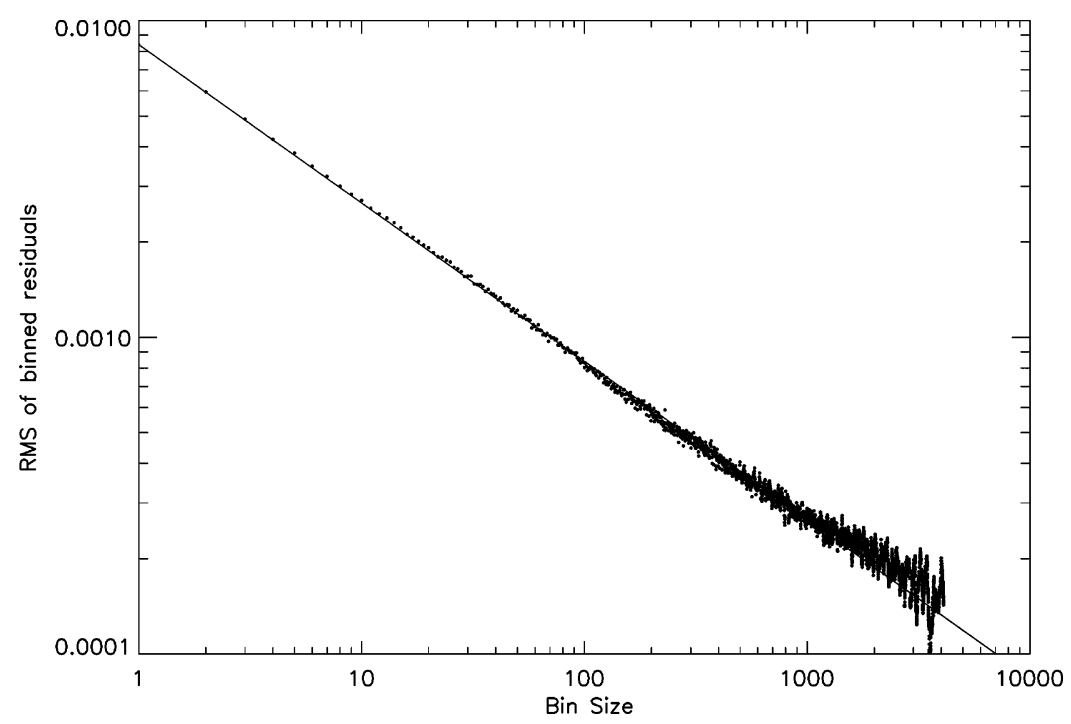

Figure 2. The root-mean-square of binned residuals vs. bin size. The solid line is proportional to $N^{-1 / 2}$. 


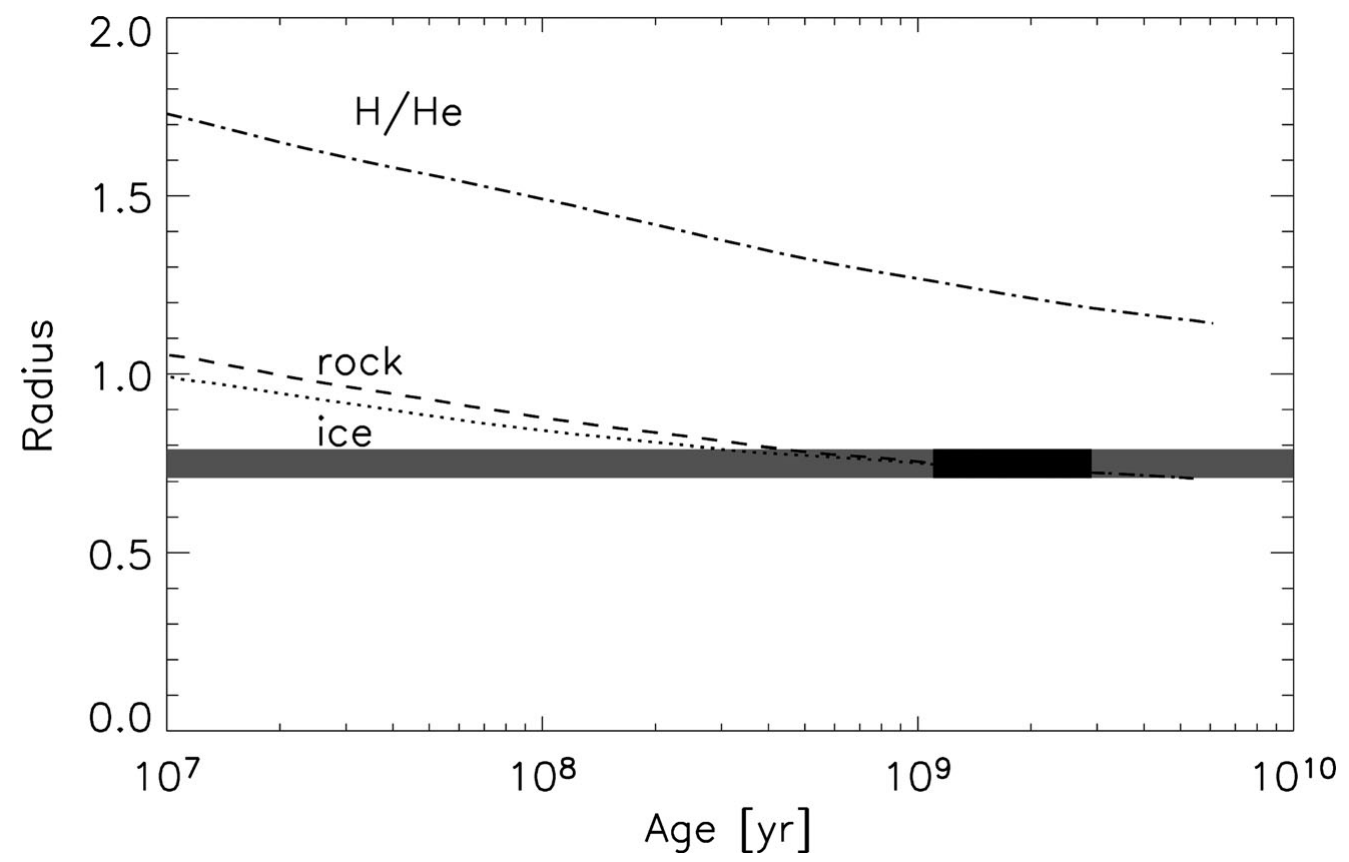

Figure 3. The radius of HD 149026b for three planet models of Fortney et al. (2006). The light grey band represents the observational radius constraint from this work. The dark grey box includes the age constraint of Sato et al. (2005). The "icy" model is for an H/He envelope and a $77 M_{\oplus}$ core of ice, while the "rocky" model is for an He/He envelope and a $65 M_{\oplus}$ core of olivine. A solar composition model (dot-dashed line), in which the planet is predominantly $\mathrm{H} / \mathrm{He}$, is excluded by the observational constraints.

\section{References}

Charbonneau, D., et al. 2006, ApJ, 636, 445

Fortney, J. J., Saumon, D., Marley, M. S., Lodders, K., \& Freedman, R. S. 2006, Nature, 642, 495

Fortney, J. J., Lodders, K., Marley, M. S., \& Freedman, R. S. 2008, ApJ, 678, 1419

Harrington, J., Luszcz, S., Seager, S., Deming, D., \& Richardson, L. J. 2007, Nature, 447, 691

Kervella, P., Thévenin, F., Di Folco, E., \& Ségransan, D. 2004, Astronomy 83 Astrophysics, 426, 297

Knutson, H. A., et al. 2007, Nature, 447, 183

Nutzman, P., Charbonneau, D., Winn, J. N., Knutson, H. A., Fortney, J. J., Holman, M. J., \& Agol, E. 2008, ArXiv e-prints, 805, arXiv:0805.0777

Pollack, J. B., Hubickyj, O., Bodenheimer, P., Lissauer, J. J., Podolak, M., \& Greenzweig, Y. 1996, Icarus, 124, 62

Sato, B., et al. 2005, ApJ, 633, 465

Winn, J. N., Henry, G. W., Torres, G., \& Holman, M. J. 2008, ApJ, 675, 1531 